\title{
Benchmark des approches d'analyse trajectographique par analyse comparative de simulations prédictives et d'essais de terrain
}

Collectif des participants à l'action A.3.1 du projet C2ROP (Antea, Arias-Montagne, Alp'Géorisques, BG21, BRGM, CD06, CD38, CD73, Céréma, Géolithe, GIA, NGE, IFSTTAR, IMSRN, INRAE, ISTERRE, 3SR, Sage, $\mathrm{SNCF}$ )

Correspondant : Franck Bourrier, Univ. Grenoble Alpes, INRAE, ETNA, 38000 Grenoble, France

- franck.bourrier@inrae.fr

\section{Résumé}

Une étude comparative de résultats d'essais de lâcher de blocs et de simulations prédictives fournies par 18 participants a été menée dans l'optique d'évaluer les capacités prédictives des études trajectographiques. La propagation d'une centaine de blocs rocheux sur deux zones de propagation, comportant des discontinuités topographiques et des configurations favorisant la propagation des blocs selon des modes assimilables à du roulement, a été analysée au niveau de zones spécifiques, dénommées écrans d'évaluation. Une variabilité significative des vitesses de passage des blocs a été mesurée au niveau des écrans d'évaluation et des distributions bimodales de ces vitesses ont été observées pour les écrans situés en aval de discontinuités topographiques.

L'analyse comparative des résultats expérimentaux et des études trajectographiques met en évidence une grande variabilité des résultats de simulation fournis et, par conséquent, de fortes incertitudes associées aux prévisions, pour ces études menées sans données de calibration préalable. Par ailleurs, les outils de simulation trajectographique s'avèrent limités en termes de modélisation des phases de propagation des blocs assimilables à du roulement sur sol meuble, notamment. Enfin, les études présentent de meilleures capacités prédictives en termes de vitesses "extrêmes " que de vitesses moyennes ou de probabilité de passage des blocs.

\section{Summary}

A comparative analysis between block propagation experiments and predictive simulations of block trajectories was conducted to evaluate the predictive capacities of block propagation analyses. Approximately one hundred blocks were released on two propagation paths with topographical discontinuities and configurations promoting block rolling. The block propagation was analysed at specific locations of the paths, called evaluation screens. A significant variability of the block velocities was measured at the screens and bimodal distributions of the velocities were observed for the screens located downhill topographical discontinuities.

The comparative analysis between the experimental results and the predictive simulations shows a large variability of the simulations results, that illustrates the uncertainties related with these predictions, done without calibration data. Specific limitations of the block propagation models were shown as regards to the modelling of block propagation similar to rolling motion on soft soils. Finally, the simulations were shown more predictive for extreme velocities than for mean ones and for block passing probabilities.

\section{Mots-clefs}

Chute de blocs, analyse trajectographique, essais de terrain, benchmark, aléa de propagation

\section{Keywords}

Rockfall, trajectory analysis, field experiments, benchmark, propagation hazard 


\section{Introduction}

La caractérisation de la propagation des blocs rocheux est un élément essentiel des études de risque de chute de blocs. Bien que l'aléa de propagation puisse être évalué qualitativement à l'aide d'approches empiriques, basées notamment sur la méthode de la ligne d'énergie (Heim, 1932), l'analyse trajectographique reste le seul outil permettant une caractérisation quantitative de la fréquence et de I'intensité de l'aléa de propagation (Volkweinn et al., 2011).

La difficulté de la modélisation de la propagation des blocs réside dans le choix d'approches constituant un compromis entre pertinence physique et applicabilité. Les deux types de modèles d'analyse trajectographique existants, modèles assimilant le bloc à un point matériel et modèles intégrant explicitement la forme du bloc (Bourrier et al , 2013), permettent d'obtenir des résultats satisfaisants suite à une calibration sur des sites bien documentés (Bourrier et al., 2012). Néanmoins, plusieurs limitations de ces modèles ont été identifiés dans la littérature, notamment la modélisation de la propagation sur des topographies complexes, présentant des replats dues à la présence de routes (Bourrier et al., 2009 ; Lambert et al., 2013), ainsi que celle de phases de propagation assimilables à du roulement du bloc, typiquement sur des sols meubles ou à faible pente (Bourrier et al., 2013 ; Spadari et al., 2012).

Plusieurs travaux de recherche ont permis la réalisation d'essais de laboratoire (e.g. Chau et al., 1998; Labiouse et Heidenreich, 2009 ; Hu et al., 2018 ; Asteriou et Tsiambaos, 2018 ; Garcia, 2020) et de terrain (e.g. Giani et al., 2004 ; Dorren et al., 2006 ; Bourrier et al., 2009 ; Bourrier et al., 2012 ; Spadari et al., 2012 ; Caviezel et al., 2019) dédiés à l'étude de la propagation des blocs. Cependant, les données issues de ces travaux ne sont pas suffisantes pour explorer, à l'échelle du versant, une diversité suffisante de configurations topographiques et géotechniques. Aussi, la calibration exhaustive d'un modèle trajectographique n'est pas envisageable à partir de ces données.

Par conséquent, lors d'une étude sur un site donné, la pertinence, la précision et la robustesse des résultats de simulation sont évalués par comparaison aux événements passés relevés sur le site ou à des événements sur des sites identifiés comme similaires. Ces comparaisons sont basées sur un nombre limité de données de terrain ne permettant généralement qu'une analyse comparative en termes de distance d'arrêt des blocs et non de cinématique de ceux-ci.

Ce travail de recherche porte sur l'analyse comparative des résultats expérimentaux et de résultats d'analyses trajectographiques, réalisées par différents opérateurs et à l'aide de différents modèles, dans l'optique d'évaluer les capacités prédictives de ces simulations. Cette étude collaborative a impliqué 19 partenaires du projet national C2ROP. Suite à la définition d'un protocole commun de benchmark, des simulations trajectographiques en aveugle ont été réalisées par plusieurs partenaires. Dans un second temps, des essais de lâcher de blocs ont été menés dans des conditions similaires à celles utilisées pour réaliser les simulations. L'analyse des essais et la comparaison avec les résultats de simulation ont été menées dans l'optique d'une analyse critique des performances des logiciels trajectographiques et de leur utilisation par les opérateurs.

\section{Description du benchmark}

a. Protocole de benchmark

Le protocole de benchmark a été construit de manière collaborative entre les partenaires de l'étude. L'objectif était de tester la qualité des prévisions issues des analyses trajectographiques sans calibration préalable sur le site d'étude. Par ailleurs, le choix a été fait de se focaliser sur l'analyse des capacités des simulations trajectographiques dans une optique de protection de linéaires et non de zonage 
réglementaire. Aussi, seules les prévisions des probabilités de passage, des trajectoires et de la cinématique des blocs ont été analysées au niveau de lignes d'évaluation correspondant à des lieux potentiels d'implantation d'ouvrages. Enfin, la sélection du site d'étude a été faite de manière à privilégier des configurations identifiées dans la littérature comme problématiques en termes de prévision des trajectoires des blocs.

Suite à la sélection du site d'étude, des conditions de lâcher des blocs ont été définies (zone de départ, formes des blocs, gamme de volume des blocs, hauteur de chute) et un Modèle Numérique de Terrain (MNT) du site a été réalisé par photogrammétrie (résolution : $0,2 \mathrm{~m}$ ).

Les simulations trajectographiques ont été menées par les participants avant que les essais n'aient eu lieu de manière à garantir des conditions de réalisation sans données préalables de calibration. Toutes les données d'entrée nécessaires à la réalisation des simulations ont été fournies aux participants : MNT, fichiers SIG de localisation des zones de départ et des zones de mesure des vitesses des blocs, conditions de lâcher des blocs, gamme de volume des blocs lâchés, localisation des lignes d'évaluation des probabilités de passage, trajectoires et cinématiques des blocs. En complément, une visite de terrain a été organisée préalablement à la réalisation des simulations par les partenaires. Chaque participant a fourni une étude trajectographique complète comprenant une description détaillée du logiciel utilisé et des conditions de réalisation des simulations ainsi que des prévisions des probabilités de passage, trajectoires et cinématiques des blocs aux niveau des lignes d'évaluation.

Une fois les résultats de simulation rendus par tous les participants, les essais ont été réalisés sur le site d'étude. Les résultats d'essai ont enfin été traités, analysés et comparés aux prévisions trajectographiques.

Initialement, deux phases supplémentaires étaient prévues : réalisation de simulations complémentaires à partir d'un échantillon réduit de résultats d'essais (de l'ordre de 5 blocs par profil) et d'un échantillon exhaustif ; mais, ces phases n'ont pas été menées à bien en raison de contraintes matérielles.

\section{b. Site d'étude}

Le site d'étude, situé dans une carrière (Authume - France, propriétaire: Pernot S.A, Figure 1), a été sélectionné en raison de sa complexité topographique et de la spécificité des caractéristiques de sols. Deux zones de propagation ont été sélectionnées de manière à privilégier des modes de propagation des blocs potentiellement difficiles à prédire. Le choix s'est porté sur des zones présentant des sols meubles associés à des pentes faibles, favorisant le déplacement des blocs en roulement, ainsi que sur des topographies complexes, présentant des ressauts et des couloirs préférentiels.

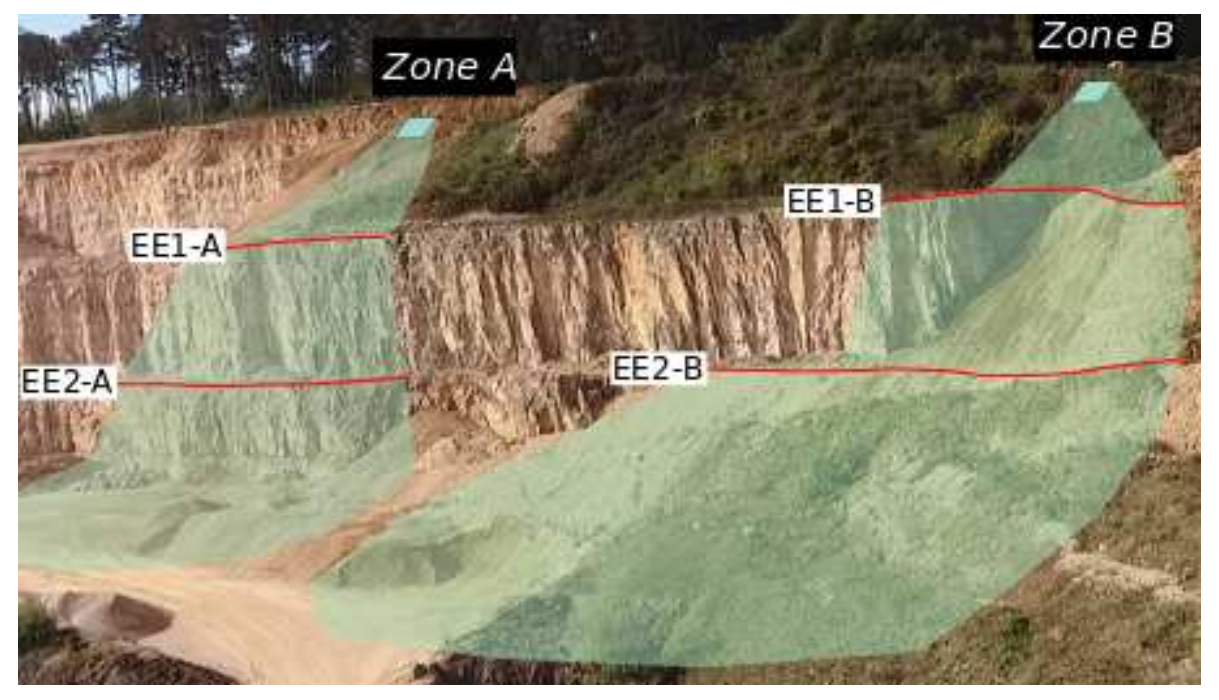


Figure 1 : Vue d'ensemble du site d'étude. Les zones de départ potentielles sont matérialisées par des carrés verts et les écrans d'évaluation par des lignes rouges.

La première zone de propagation (Zone A - Figure 1) se caractérise par une pente amont faible (de l'ordre de $32^{\circ}$ ), constituée de sols très meubles, surplombant deux ressauts topographiques successifs séparés par un replat court. Le second ressaut est situé en amont d'une grande zone de replat. La seconde zone de propagation (Zone B - Figure 1) est également caractérisée par une pente amont faible constituée de sols meubles sur un dénivelé d'environ $15 \mathrm{~m}$ surplombant un goulot d'étranglement séparant une falaise et une pente de sols meubles similaire à la pente amont mais orientée selon une direction différente. La partie aval de cette zone est constituée de deux pistes légèrement inclinées surplombant une pente de $32^{\circ}$ de sols meubles.

Ces deux zones présentent des caractéristiques topographiques différentes. Le profil de la zone $A$ est relativement uniforme, ce qui suggère une propagation des blocs majoritairement bidimensionnelle alors que la complexité de la topographique de la zone B favorise la présence d'un couloir de propagation préférentiels. Par ailleurs, sur la zone A, l'effet de discontinuités topographiques marquées sur les trajectoires des blocs pourra être clairement étudié en raison de la succession de deux parois verticales de $18 \mathrm{~m}$ et $12 \mathrm{~m}$ alors que, sur la zone $\mathrm{B}$, l'accent est mis sur l'effet de changements de pente et d'orientation successifs. Enfin, sur les deux zones, la présence de pentes faibles, combinées à des sols meubles permettra de focaliser l'étude sur la propagation des blocs selon un mode assimilable à du roulement.

\section{c. Essais de lâcher de blocs}

Environ 50 blocs ont été lâchés successivement sur chaque zone de propagation à l'aide d'une pelle mécanique. Les blocs utilisés pour la campagne d'essais ont été sélectionnés visuellement sur le site $d^{\prime}$ étude de manière à obtenir une gamme de volume comprise entre $0,1 \mathrm{~m}^{3}$ et $0,75 \mathrm{~m}^{3}$. Avant les essais, tous les blocs ont été pesés à l'aide d'un capteur de force piézo-résistif (Chatillon SLC10000 - précision : 20kg). Préalablement aux essais de chute de blocs, une zone de lâcher des blocs $(4 \mathrm{~m} \times 4 \mathrm{~m})$ a été définie pour chaque zone de propagation. Lors d'essais préliminaires, une hauteur minimale de lâcher des blocs garantissant la propagation des blocs sur la partie amont a été définie. La hauteur de lâcher des blocs a ainsi été fixée à $4 \mathrm{~m}$ pour la zone $\mathrm{A}$ et $2 \mathrm{~m}$ pour la zone $\mathrm{B}$.

La propagation de chaque bloc a été filmée à l'aide de plusieurs caméras. L'analyse des films s'est focalisée sur la caractérisation de la cinématique des blocs au niveau de zones spécifiques, dénommées écrans d'évaluation (Figure 1). Pour la zone $A$, un premier écran d'évaluation (EE1-A) a été positionné au niveau du ressaut supérieur et un second écran (EE2-A) au niveau du ressaut intermédiaire. Pour la zone $B$, le premier écran d'évaluation (EE1-B) a été positionné le long de la ligne de niveau passant par le goulot d'étranglement et le second écran (EE1-B) à l'extrémité amont de la piste supérieure.

Les vitesses de translation, de rotation et les hauteurs de passage des blocs ont été caractérisées au niveau de chaque écran d'évaluation à partir des films des trajectoires.

Pour quantifier les vitesses de translation, deux positions de chaque bloc (juste avant et juste après le passage de l'écran d'évaluation) ont tout d'abord été identifiées; puis, la vitesse de translation du bloc a été calculée à partir des positions de ces points et du temps de trajet du bloc entre les deux points. Pour un bloc passant au niveau de l'écran avec une hauteur suffisante, les positions des blocs avant et après l'écran correspondent à des points d'impact. Aussi, la vitesse du bloc au niveau de l'écran a été calculée à partir des équations caractérisant le vol parabolique d'un objet. Dans le cas contraire, la vitesse moyenne entre les deux positions du bloc a été calculée. La détermination des positons des blocs en 3D a été faite de façon 
similaire dans les deux cas. L'image du film associé à chaque position a été projetée sur le MNT, permettant l'identification des coordonnées du point.

Les vitesses de rotation ont été estimées visuellement par comptage du nombre de tour réalisés par le bloc entre les deux positions et calcul d'une vitesse de rotation moyenne. Finalement, la hauteur de passage du centre de gravité du bloc a été estimée qualitativement à partir des films. Quatre classes sont définies : contact avec le sol (hauteur inférieure à $0,5 \mathrm{~m}$ ), faible hauteur (comprise entre $0,5 \mathrm{~m}$ et $1 \mathrm{~m}$ ), hauteur moyenne (comprise entre $1 \mathrm{~m}$ et $3 \mathrm{~m}$ ), hauteur importante (supérieure à $3 \mathrm{~m}$ ).

\section{Résultats}

a. Essais

La description des résultats expérimentaux se focalise sur la présentation des données essentielles à l'analyse des résultats de simulation. Une description détaillée de ces résultats expérimentaux peut être consultée dans (Bourrier et al., 2020).

Pour la zone $A$, les vitesses de translation des blocs au niveau de l'écran d'évaluation 1 (EE1-A) varient sur une large gamme (de 0,1 à $8,9 \mathrm{~m} / \mathrm{s}$ - Figure 2). Leur distribution est globalement continue mais elle n'est pas symétrique : $50 \%$ des blocs passent avec une vitesse inférieure à $2 \mathrm{~m} / \mathrm{s}$ alors que $10 \%$ des vitesses sont supérieures à $5 \mathrm{~m} / \mathrm{s}$. La distribution des vitesses au niveau de l'écran d'évaluation 2 (EE2-A) présente un caractère bimodal marqué : $32 \%$ des vitesses sont inférieures à $5 \mathrm{~m} / \mathrm{s}$ et $68 \%$ d'entre elles sont comprises entre $18 \mathrm{~m} / \mathrm{s}$ et $19,5 \mathrm{~m} / \mathrm{s}$.

Pour la zone B, la gamme de variation des vitesses de translation est plus faible au niveau d'EE1-B qu'au niveau d'EE1-A et la distribution des vitesses est quasiment symétrique à l'exception de quelques blocs se propageant à faible vitesse (vitesses inférieure à $2,5 \mathrm{~m} / \mathrm{s}$ pour $6 \%$ des blocs). Au niveau de l'écran d'évaluation 2 (EE2-B), la distribution présente un caractère bimodal mais celui-ci est largement moins marqué qu'au niveau d'EE2-A : $71 \%$ des vitesses sont comprises entre $0 \mathrm{~m} / \mathrm{s}$ et $5 \mathrm{~m} / \mathrm{s}$ et $29 \%$ entre $6 \mathrm{~m} / \mathrm{s}$ et $14 \mathrm{~m} / \mathrm{s}$.

Le Tableau 1 fournit des indicateurs statistiques des distributions des vitesses au niveau des écrans d'évaluation. Ces indicateurs confirment que les distributions ne sont pas symétriques. En effet, les valeurs moyennes sont très différentes des valeurs médianes. Par ailleurs, les écart-types des distributions des vitesses sont élevés comparativement aux valeurs moyennes correspondantes, ce qui induit de forts coefficients de variation (excepté pour EE1-B) et illustre ainsi la grande variabilité des vitesses.

Les vitesses de rotation sont distribuées de façon homogène sur une gamme de valeur allant de $3 \mathrm{rad} / \mathrm{s}$ à 20rad/s. Elles sont globalement plus faibles pour la zone A que pour la zone B et leur variabilité est plus faible que celle des vitesses de translation (coefficients de variation plus faibles - Tableau 1).

L'analyse qualitative des hauteurs de passage met en évidence une tendance des blocs à se propager à faible hauteur au niveau des écrans d'évaluation. Pour la zone $A$, tous les blocs passent au niveau de l'écran EE1-A en contact avec le sol et seulement quelques-uns d'entre eux passent au niveau de l'écran EE2-A avec des hauteurs moyennes ou importantes. La faible quantité de blocs passant au niveau de l'écran EE2-A avec une hauteur importante n'est à première vue pas cohérente avec les vitesses de passage des blocs importantes au niveau de cet écran. Cette incohérence apparente est due au fait que plusieurs blocs ont impacté le bloc juste au niveau de l'écran EE2-A. Pour ces blocs, nous avons considéré leur vitesse de passage juste avant impact dans l'analyse car cela s'avère conservatif en termes de dimensionnement d'ouvrage. Pour la zone B, tous les blocs, à l'exception d'un, sont passés au niveau des deux écrans d'évaluation en contact avec le sol ou à faible hauteur (inférieure à $1 \mathrm{~m}$ ). 


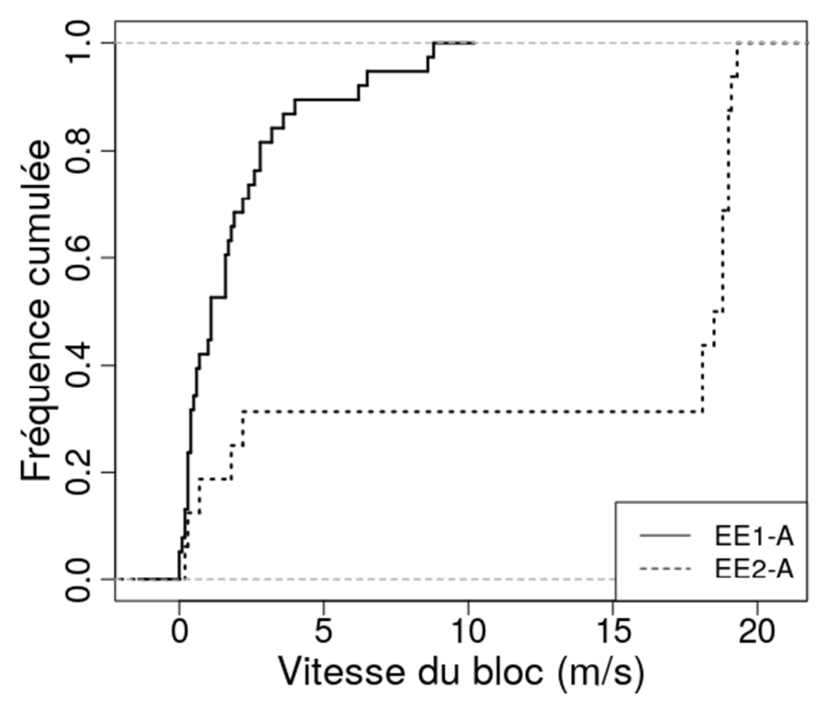

a)

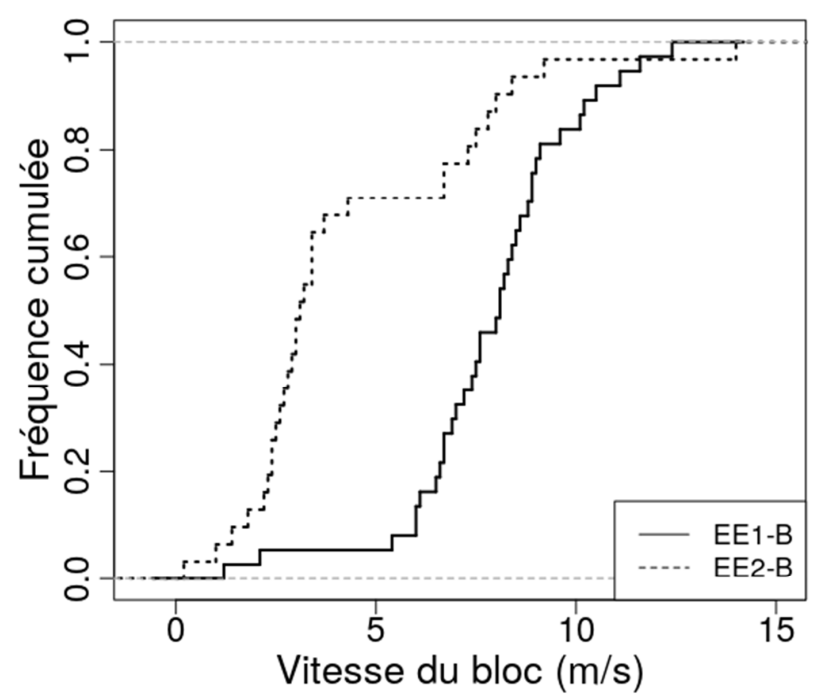

b)

Figure 2 : Fonctions de répartition des vitesses des blocs au niveau des écrans d'évaluation pour les zones $A$ (a) et B (b).

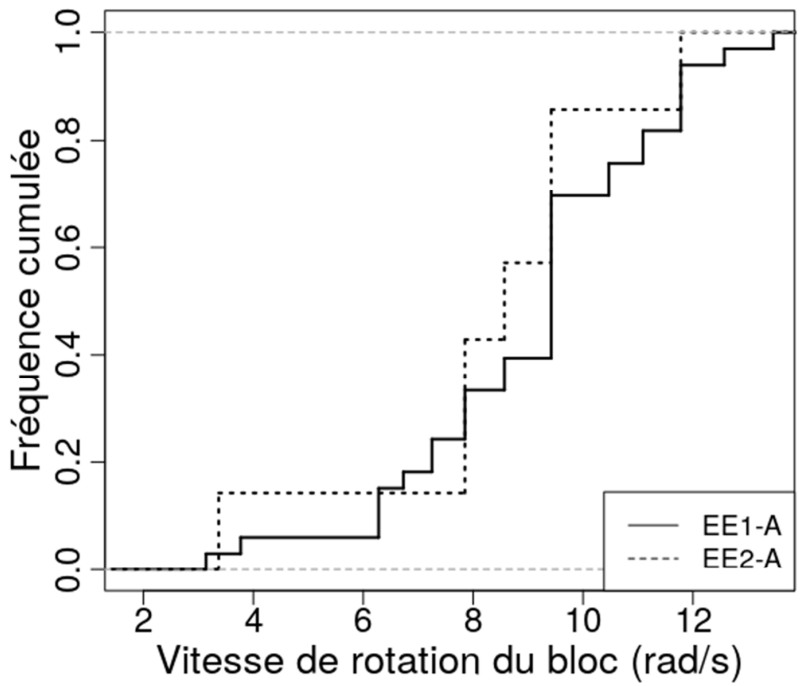

a)

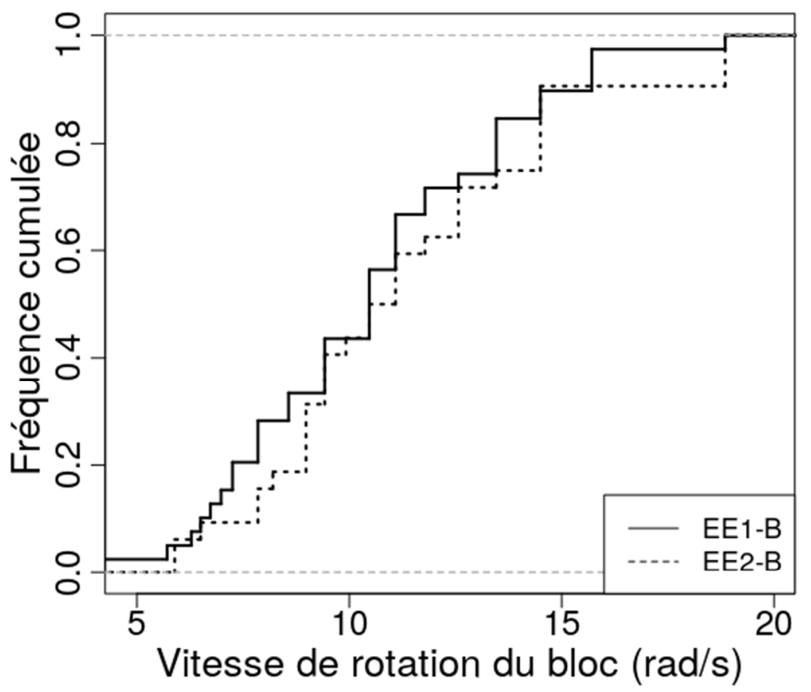

b)

Figure 3 : Fonctions de répartition des vitesses de rotation des blocs au niveau des écrans d'évaluation pour les zones A (a) et B (b).

\begin{tabular}{|c|c|c|c|c|c|}
\hline & & \multicolumn{2}{|c|}{ Zone A } & \multicolumn{2}{|c|}{ Zone B } \\
\hline & & EE1-A & EE2-A & EE1-B & EE2-B \\
\hline & Passage des blocs (\%) & 96 & 41 & 93 & 82 \\
\hline \multirow{4}{*}{ Vitesse } & Moyenne $(\mathrm{m} / \mathrm{s})$ & 1,9 & 13,2 & 7,9 & 4,2 \\
\hline & Médiane $(\mathrm{m} / \mathrm{s})$ & 1,1 & 18,6 & 8,1 & 3,1 \\
\hline & Ecart-type $(\mathrm{m} / \mathrm{s})$ & 8,5 & 8,5 & 2,2 & 3,0 \\
\hline & Coeff. Var. (\%) & 447 & 64 & 28 & 71 \\
\hline \multirow{4}{*}{$\begin{array}{l}\text { Vitesse de } \\
\text { rotation }\end{array}$} & Moyenne (rad/s) & 9,0 & 8,0 & 10,5 & 11,3 \\
\hline & Médiane (rad/s) & 9,4 & 8,2 & 10,5 & 10,8 \\
\hline & Ecart-type (rad/s) & 2,4 & 2,5 & 3,3 & 3,5 \\
\hline & Coeff. Var. (\%) & 26 & 31 & 31 & 31 \\
\hline
\end{tabular}




\begin{tabular}{r|r|c|c|c|c}
\hline & $\mathrm{h}<0,5 \mathrm{~m}(\%)$ & 100 & 65 & 84 & 70 \\
\cline { 2 - 6 } Hauteur de & $0,5 \mathrm{~m}<\mathrm{h}<1 \mathrm{~m}(\%)$ & 0 & 25 & 14 & 30 \\
\cline { 2 - 6 } passage (h) & $1 \mathrm{~m}<\mathrm{h}<3 \mathrm{~m}(\%)$ & 0 & 5 & 2 & 0 \\
\cline { 2 - 6 } & $\mathrm{h}>3 \mathrm{~m}(\%)$ & 0 & 5 & 0 & 0
\end{tabular}

Tableau 1 : Tableau de synthèse des résultats expérimentaux

\section{b. Simulations}

$\mathrm{Au}$ total, 18 résultats de simulation ont été obtenus. Dix sociétés ou institutions ( 6 bureaux d'études, 3 instituts de recherche, 1 laboratoire universitaire) ont fourni des résultats de simulation. Certains partenaires ont fourni plusieurs résultats issus des travaux de différents experts.

Ces résultats ont été obtenus à l'aide de différents outils :

- 10 partenaires ont utilisé des logiciels trajectographiques 2D basés sur une approche de type "point matériel ", c'est-à-dire utilisant des modèles de rebond permettant de modifier la vitesse du bloc au niveau du centre de gravité à l'aide de coefficients de restitution. Parmi ces études, 3 ont été réalisées avec le même logiciel.

- 1 partenaire a utilisé un logiciel trajectographique 2D permettant de modéliser explicitement la forme du bloc. Dans cette approche, des forces de contact sont appliquées au bloc au cours de l'interaction avec le terrain naturel au niveau de points de contacts.

- 6 partenaires ont utilisé des logiciels trajectographiques 3D basés sur une approche de type « point matériel ». Parmi ces études, 4 ont été réalisées avec le même logiciel.

- 1 partenaire a utilisé un logiciel trajectographique 3D permettant de modéliser explicitement la forme du bloc.

Chaque partenaire a fourni une description des principes du modèle utilisé pour l'étude. Cependant, les descriptions étaient généralement très succinctes concernant les éléments théoriques justificatifs du modèle, l'implémentation numérique, les critères d'arrêt des simulations, et les paramètres du modèle, notamment. Par ailleurs, les résultats fournis ne comportaient pas plusieurs éléments essentiels à l'évaluation de la pertinence des simulations. Les éléments majeurs généralement manquants étaient l'analyse de la convergence des simulations, des éléments quantitatifs justifiant la calibration du modèle, la description des valeurs de tous les paramètres du modèle de rebond, la description détaillée des conditions initiales de lâcher des blocs et des propriétés des blocs. Au contraire, des descriptions détaillées des choix de "types de sol " et de volumes des blocs utilisés pour réaliser les simulations ont généralement été fournies. Enfin, les résultats de simulation fournis étaient généralement trop succincts pour permettre une analyse statistique complète. Les résultats fournis se limitaient généralement à une présentation d'indicateurs statistiques des distributions (moyennes, médianes, quantiles, maximum,...) sans préciser leur mode de calcul. Les distributions complètes des vitesses et hauteurs de passage au niveau des écrans d'évaluation n'ont été fournies que par une minorité de participants.

Au vu des résultats des essais et des rendus de simulation, nous avons établi un protocole d'analyse des essais s'appuyant essentiellement sur la comparaison d'indicateurs statistiques obtenus au niveau des écrans de passage des blocs. Les pourcentages de passage des blocs et les indicateurs statistiques des distributions des vitesses au niveau des écrans ont été comparés de façon quantitative, en particulier. Bien que les différents partenaires aient transmis des données hétérogènes s'agissant des indicateurs statistiques des distributions, ils ont tous fourni à la fois des indicateurs représentatifs des valeurs " moyennes" des distributions (moyennes ou médianes) et des valeurs " extrêmes " des distributions (quantile à $95 \%$, quantile à $99 \%$, maximum,...). Nous avons fait le choix de focaliser notre analyse sur la 
comparaison de ces indicateurs "moyens" et "extrêmes", bien qu'ils soient différents selon les partenaires, afin de garantir un nombre suffisant de données à comparer. Par ailleurs, le choix a été fait de réaliser une analyse commune pour tous les types de logiciel utilisés (2D et 3D, « point matériel » et « prise en compte de la forme ") car nous ne disposons pas d'un nombre suffisant de simulations pour tous les types de logiciels.

Les probabilités de passage prédites par les participants (Figure 4) se répartissent sur une large gamme de variation, supérieure à $50 \%$ quel que soit l'écran de passage. Etant donné la grande étendue de cette plage de variation, les valeurs expérimentales se situent pour tous les écrans, à l'exception d'EE1-A, entre le premier et le troisième quartile de la distribution des probabilités prédites. Pour l'écran EE1-A, la valeur expérimentale est supérieure aux valeurs majoritairement prédites par les participants.

Pour la zone A, la moyenne et la médiane des vitesses mesurées sont différentes des vitesses « moyennes » majoritairement prédites (Figure 5). En effet, la plage de variation des vitesses prédites ne couvre ni la moyenne ni la médiane. Ces valeurs sont surestimées par les simulations pour l'écran EE1-A et sousestimées pour l'écran EE2-A. Les prévisions des vitesses « moyennes » sont en meilleure adéquation avec la moyenne et la médiane des vitesses mesurées pour la zone B. Ces valeurs sont situées entre le premier et le troisième quartile de la distribution des vitesses prédites pour l'écran EE1-B et seule la médiane des vitesses est surestimée pour l'écran EE2-B.

Contrairement aux autres quantités prédites, les vitesses « extrêmes " prédites se répartissent sur une gamme de variation plus restreinte (Figure 6). La vitesse maximale mesurée se trouve entre le premier et le troisième quartile des distributions des vitesses "extrêmes " prédites pour tous les écrans, sauf pour l'écran EE2-B pour lequel cette valeur est supérieure aux vitesses « extrêmes » prédites. Le quantile 0,95 des distributions des vitesses mesurées est similaire aux vitesses « extrêmes " prédites pour les écrans EE2A et EE1-B alors que cette valeur est surestimée pour les écrans EE1-A et EE2-B.

En accord avec l'analyse qualitative des hauteurs de passage des blocs, les hauteurs prédites sont faibles, qu'elles soient « moyennes » ou « maximales " (Figure 7 et 8). Il est cependant intéressant de noter que ces valeurs sont légèrement plus élevées (de l'ordre de 1 à $2 \mathrm{~m}$ ) que celles observées dans les essais (généralement inférieures à $1 \mathrm{~m}$ ). Des hauteurs de passage "extrêmes " plus élevées sont tout de même prédites pour l'écran EE2-A, seul écran pour lequel un bloc passant à une hauteur supérieure à $3 \mathrm{~m}$ a été observé. 


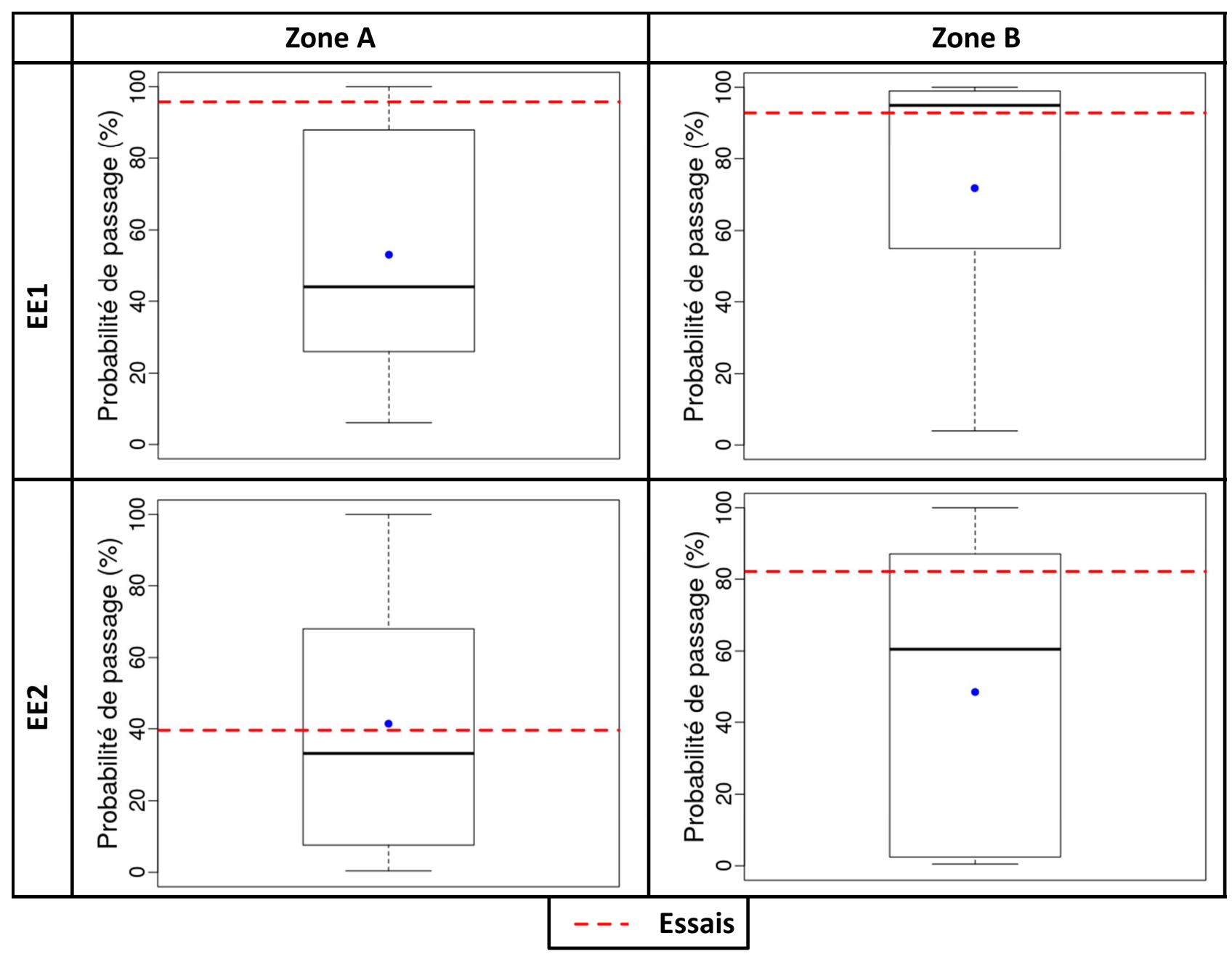

Figure 4 : Diagramme en boîte ${ }^{1}$ représentant la distribution des probabilités de passage fournies par les participants. Le point bleu représente la moyenne de cette distribution et la ligne rouge (trait pointillé) est associée à la valeur obtenue lors des essais.

\footnotetext{
${ }^{1}$ Dans ce type de représentation, les extrémités inférieures et supérieures de la boîte correspondent aux $1^{\text {er }}$ et $3^{\text {è }}$ quartiles de la distribution. La médiane de la distribution est matérialisée par le trait gras à l'intérieur de la boite. Le segment inférieur (resp. supérieur) hors des boites est soit associé au minimum (resp. maximum) soit situé à une distance de 1,5 fois la hauteur de la boite au-dessous (resp. au-dessus) de la boîte. Les cercles blancs représentent les valeurs de l'échantillon situées hors des limites des boîtes.
} 


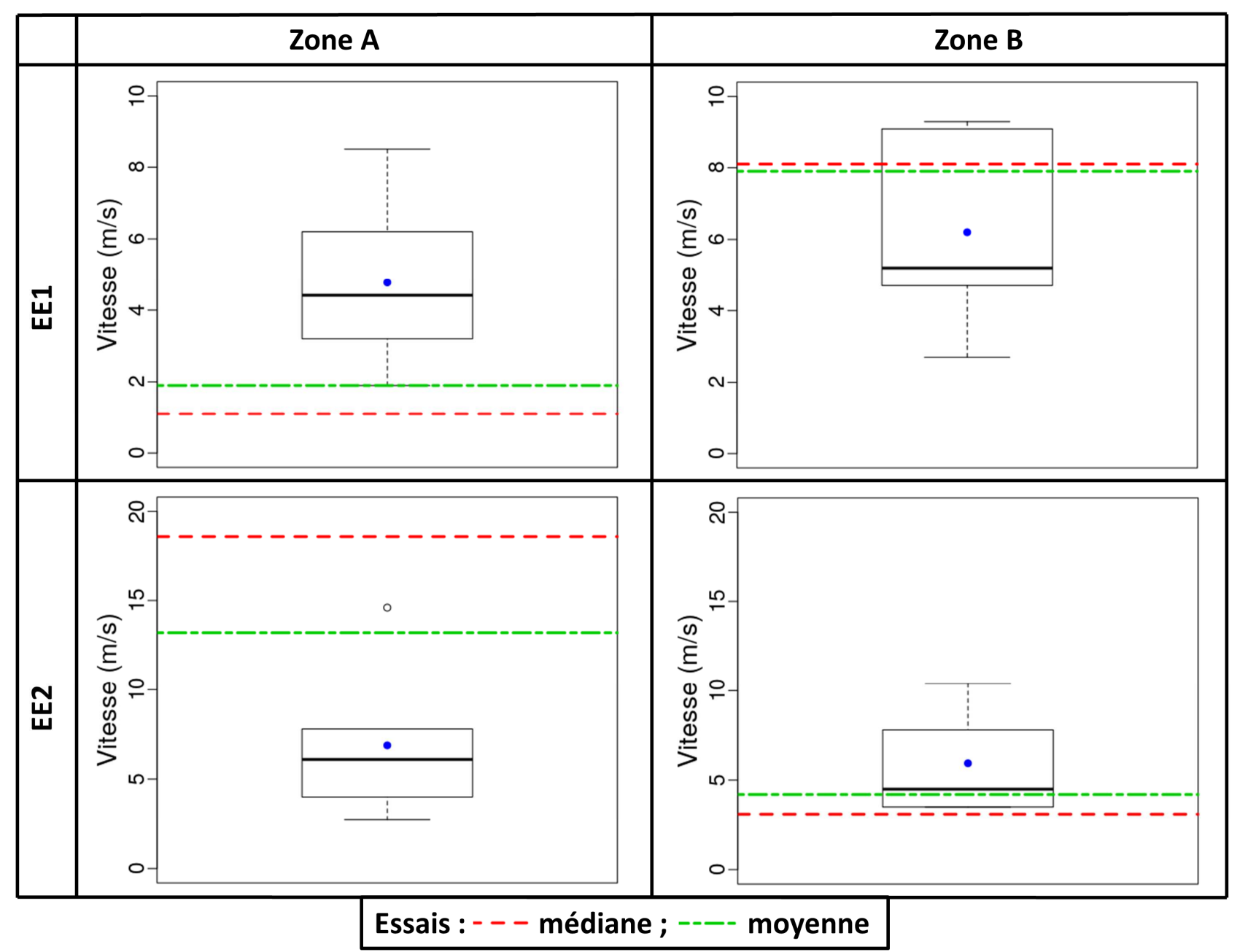

Figure 5 : Diagramme en boîte ${ }^{1}$ représentant la distribution des vitesses « moyennes » fournies par les participants. Le point bleu représente la moyenne de cette distribution, les lignes rouges et vertes (respectivement en trait pointillé et trait mixte) sont associées à la médiane et à la moyenne de la distribution des vitesses issues des essais. 


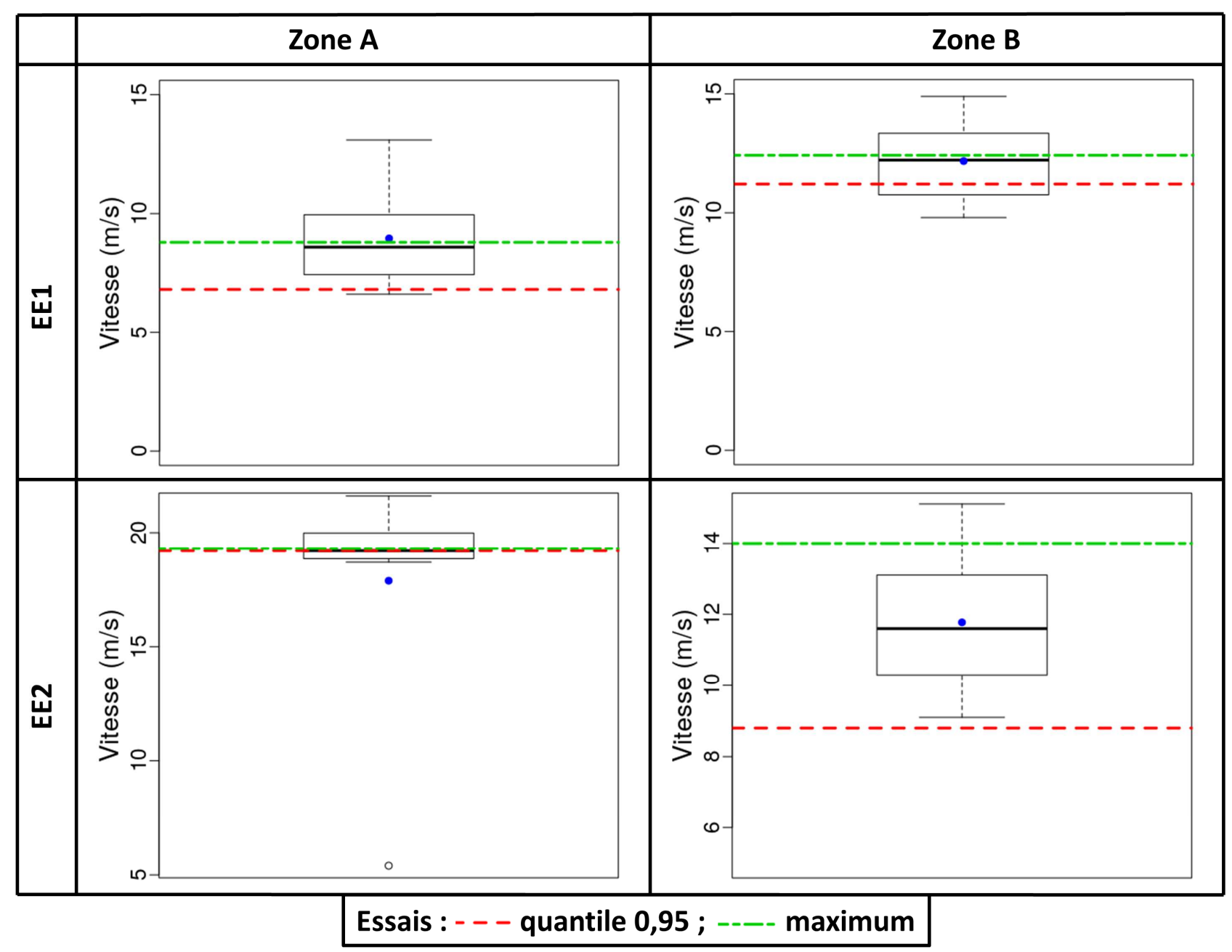

Figure 6 : Diagramme en boîte ${ }^{1}$ représentant la distribution des vitesses «extrêmes » fournies par les participants. Le point bleu représente la moyenne de cette distribution, les lignes rouges et vertes (respectivement en trait pointillé et trait mixte) sont associées au quantile 0,95 et au maximum de la distribution des vitesses issues des essais. 


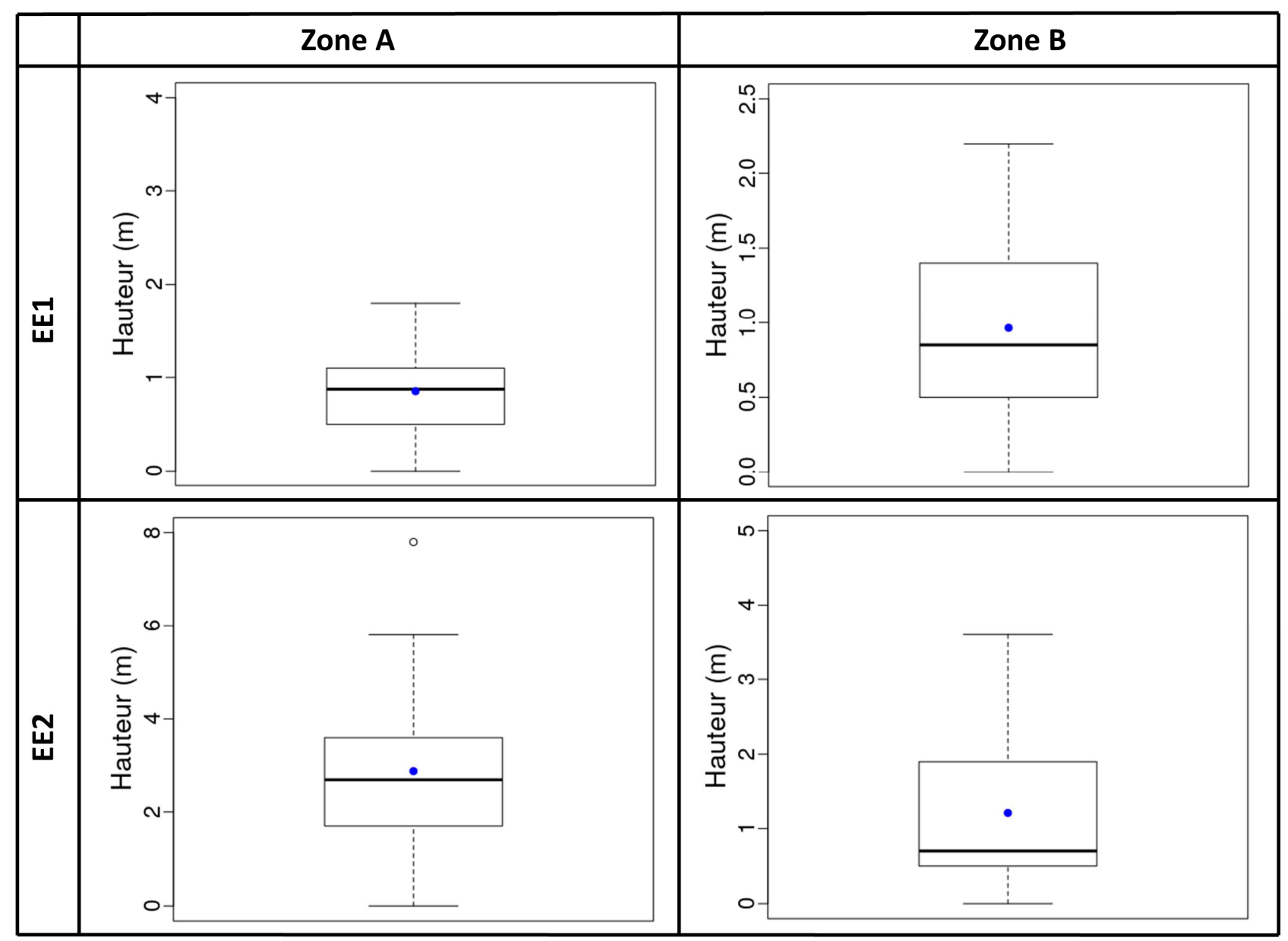

Figure 7 : Diagramme en boîte ${ }^{1}$ représentant la distribution des hauteurs « moyennes » fournies par les participants. Le point bleu représente la moyenne de cette distribution. 


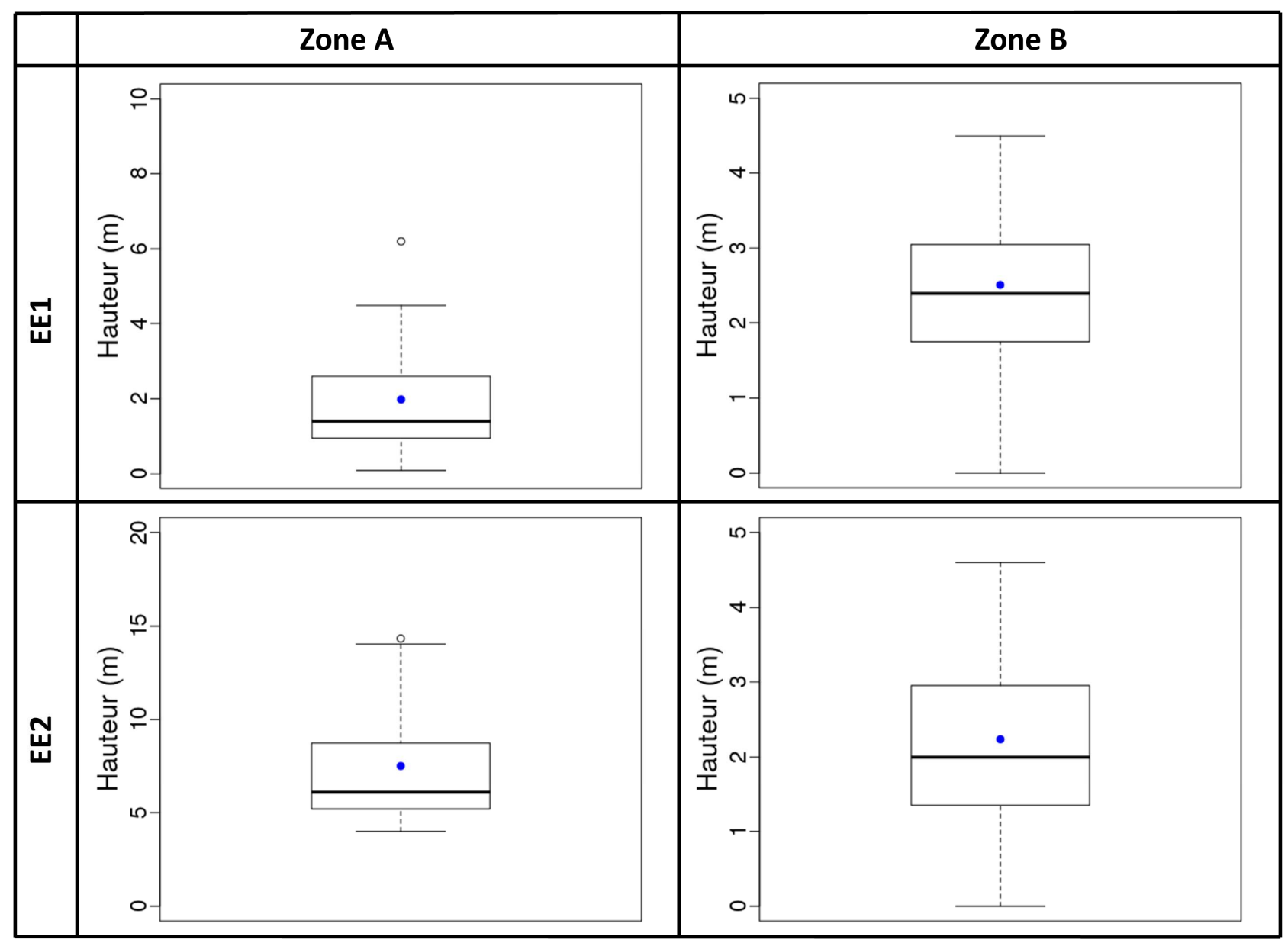

Figure 7 : Diagramme en boîte ${ }^{1}$ représentant la distribution des hauteurs " extrêmes » fournies par les participants. Le point bleu représente la moyenne de cette distribution.

\section{Analyse critique}

La propagation des blocs sur la zone A se caractérise par un mouvement proche du sol, assimilable à du roulement, à faible vitesse, jusqu'à l'écran d'évaluation EE1-A. Peu d'arrêts des blocs ont été observés sur la partie supérieure du profil. Au niveau de l'écran 1, deux modes de franchissement ont été observés. Le premier mode induit un impact vers le milieu du replat intermédiaire suivi soit d'un arrêt sur ce replat soit d'un passage de l'écran EE2-A à faible vitesse. Le second mode est caractérisé par un impact, juste au niveau de l'écran EE2-A, de blocs arrivant directement du ressaut amont à haute vitesse. Sur la zone $B$, tous les blocs se déplacent selon un mode de propagation assimilable à du roulement jusqu'à l'écran EE1-B. La plupart d'entre eux franchit l'écran EE1-B au niveau du goulot d'étranglement, à l'exception de deux blocs ayant dévié vers l'affleurement rocheux attenant. Les trajectoires des blocs ne deviennent significativement différentes qu'à partir de la discontinuité topographique induite par la piste amont (changement de pente). Ces différences induisent une distribution bimodale des vitesses des blocs au niveau de l'écran EE2-B, due comme pour l'écran EE2-A, à l'existence de mode de propagation des blocs sur la piste, soit par succession de rebonds rapprochés soit par un nombre réduit de rebonds plus éloignés. L'occurrence d'un mode de propagation ou de l'autre semble fonction de l'orientation du bloc lors du premier impact sur la piste, essentiellement.

A priori, la prévision de la propagation des blocs jusqu'aux écrans d'évaluation amont (EE1-A et EE1-B) peut paraitre simple. En effet, le profil topographique est régulier, le type de sol est homogène, les conditions de 
départ sont clairement définies ainsi que les masses et formes des blocs. En dépit de cette apparente simplicité, les prévisions fournies par les participants s'avèrent très variables, notamment en termes de probabilités de passage et de vitesses « moyennes ». Au contraire, la variabilité des prévisions des vitesses " extrêmes " est bien plus réduite, rendant leur utilisation pratique envisageable. Par ailleurs, pour les vitesses " extrêmes ", les valeurs mesurées expérimentalement appartiennent à la gamme de variation des valeurs prédites pour EE1-A et EE1-B alors que, pour les probabilités de passage et les vitesses "moyennes», ce n'est le cas que pour EE1-B. Les différences notables observées pour l'écran EE1-A s'expliquent par le fait que la propagation sur la zone de propagation amont du profil A est plus complexe à prédire. En effet, la faible pente du terrain combinée à un sol très meuble conduit à une configuration favorisant la propagation des blocs par une succession de petits rebonds rapprochés, un mode de propagation proche du roulement, associé à une cratérisation marquée du sol meuble. Cette complexité conduit à de fortes surestimations des vitesses de propagation sur la partie amont de la zone $A$, ce qui met en évidence les difficultés des logiciels à traduire la propagation des blocs à faible vitesse dans un mode proche du roulement sur des sols meubles. Deux raisons peuvent expliquer ces difficultés de modélisation. Tout d'abord, la cratérisation marquée du sol induit des processus d'interaction complexes lors du rebond, plastification significative $d u$ sol et création d'un bourrelet en aval du bloc notamment, qui sont généralement pris en compte de façon très simplifiée dans les outils trajectographiques. Par ailleurs, la cinématique des blocs selon ce mode de propagation est difficile à traduire par les outils trajectographiques. Pour les modèles de type "point matériel », ce mode de propagation se situe à la frontière entre les deux types de propagation les plus classiquement intégrés: la succession de rebonds éloignés et de phases de vol et le roulement. Cependant, aucun de ces modes de propagation n'est réellement adapté dans ce cas. Les outils intégrant explicitement la forme du bloc sont a priori plus adaptés. Néanmoins, I'hypothèse, généralement faite dans ces outils, d'un point de contact unique n'évoluant pas au cours de l'interaction est certainement très limitante pour modéliser ce mode de propagation dans lequel la localisation du point de contact évolue au cours du contact avec le sol.

Au niveau des écrans d'évaluation EE2-A et EE2-B, on observe une diversité importante des modes de propagation des blocs ainsi que des distributions bimodales des vitesses des blocs, notamment en raison de la présence de différents modes de franchissement des discontinuités topographiques en amont de ces écrans d'évaluation. Les distributions bimodales des vitesses peuvent être interprétées comme étant des combinaisons de deux distributions de vitesses associées à deux modes de franchissement de la discontinuité directement en amont des lignes d'évaluations EE2. La prévision de la distribution complète des vitesses au niveau des EE2 nécessite donc d'identifier les différents modes de franchissement des discontinuités, ainsi que leur occurrences relatives. Même si la distribution des vitesses avant le franchissement de la discontinuité est connue avec une incertitude raisonnable, il est donc très difficile de prédire la distribution complète des vitesses au niveau des EE2. Il est plus raisonnable de quantifier seulement les vitesses " extrêmes » au niveau de EE2-B car cela nécessite " seulement» de prédire le mode de franchissement induisant les vitesses les plus importantes et de quantifier ces vitesses " extrêmes ". Les meilleures capacités prédictives des simulations en termes de vitesses " extrêmes " tendent à confirmer cette analyse.

De manière générale, l'analyse comparative des prévisions des participants et des résultats expérimentaux permet d'évaluer la pertinence des informations fournies par les études trajectographiques, les niveaux d'incertitude associés aux résultats, ainsi que les limitations de ces études. En premier lieu, les modèles de simulation trajectographique ne permettent pas une traduction suffisamment complète des processus en jeu pour traduire la propagation des blocs pour certains modes de propagation. Notamment, cette étude met en évidence que, si ces modèles semblent suffisants pour traduire la propagation des blocs lors de propagations par succession de rebonds éloignés, leur complexité demeure insuffisante pour traduire la 
propagation des blocs à faible vitesse dans un mode proche du roulement sur des sols meubles. La très grande variabilité des prévisions fournies par les participants illustre également la difficulté de réaliser des prévisions avec un niveau d'incertitude raisonnable en l'absence de données de " comparaison». Dans le cadre de cette étude, aucune donnée de comparaison n'a été fournie, ce qui a induit une grande variabilité des résultats fournis par les participants. Néanmoins, on peut supposer que, si des données de calibration avaient été fournies aux participants, même en nombre réduit, la variabilité des prévisions aurait été moindre. Les variations des capacités prédictives au niveau des différents écrans d'évaluation met également en exergue le fait que les capacités de prévision sont conditionnées par la complexité du site, qu'elle soit topographique (discontinuité, effets 3D,...) ou liée aux matériaux (présence de sols meubles, de rugosité,...). Ainsi, plus un site cumule de facteurs augmentant sa complexité, plus il devient difficile de prédire les trajectoires avec un degré de certitude suffisant. Enfin, l'analyse comparative des résultats d'essais et des prévisions met en évidence que, dans le contexte de l'étude, les simulations trajectographiques ne sont réellement prédictives que pour la prévision de l'intensité maximale de l'aléa. En effet, une telle prévision ne nécessite que d'identifier le mode de propagation des blocs conduisant aux intensités les plus élevées et de modéliser correctement ce mode. La prévision correcte de la fréquence et de l'intensité moyenne de l'aléa est largement plus complexe car elle nécessite de prévoir finement la répartition des arrêts des blocs avant l'écran et des modes de propagation (passage au-dessus du replat intermédiaire ou rebond sur ce replat).

L'analyse détaillée des études trajectographiques fournies permet enfin d'identifier plusieurs pistes pour améliorer la réalisation, la présentation et l'analyse des études trajectographiques. Tout d'abord, il est indispensable d'utiliser des logiciels bien documentés, non seulement en termes d'utilisation pratique mais surtout de processus modélisés et d'approches de modélisation. Les outils pour lesquels il existe des exemples variés d'études sur des sites bien documentés, voire des preuves de calibration, sont à privilégier. La possibilité de fournir des sorties exhaustives des données de simulation est enfin une condition nécessaire par la réalisation d'analyses statistiques détaillées. Par ailleurs, devant la forte variabilité des résultats de simulation fournis, il semble nécessaire de mettre en œuvre des campagnes de simulation plus complètes permettant de balayer les différentes situations de propagation potentielles sur le site. Dans cette optique, la réalisation de plusieurs simulations à l'aide d'outils ou d'approches de modélisation différentes sur un même site et la réalisation d'études de la sensibilité des modèles au paramétrage constituent deux pistes intéressantes. L'analyse de tels travaux peut être très informative en termes d'identification des différents processus de propagation des blocs et de leurs vraisemblances ainsi que d'évaluation des incertitudes associées aux résultats de simulations.

\section{Conclusion}

Les résultats expérimentaux fournis par l'analyse des essais de lâcher de blocs sur les deux zones de propagation du site d'étude a mis en évidence la complexité des processus de propagation sur le site. Cette complexité est notamment liée à la présence de sols meubles situés dans des zones de pentes faibles, entrainant une propagation des blocs à faible vitesse selon un processus assimilable à du roulement, et de discontinuités topographiques, franchies par les blocs selon différents modes de propagation.

L'analyse comparative des résultats de ces essais et des études trajectographiques de prévision de la propagation des blocs sur les deux zones fournies par les participants a mis en évidence une capacité prédictive plus importante des études en termes de vitesses extrêmes des blocs. Cette analyse a également permis d'identifier les limitations des outils trajectographiques en termes de modélisation de la propagation des blocs lors des phases assimilables à du roulement sur sol meuble. Par ailleurs, dans le 
contexte de cette étude sans données de calibration préalables, la grande variabilité des résultats de simulations fournis met en exergue les fortes incertitudes associées aux prévisions, notamment suite au franchissement de discontinuités topographiques.

L'intérêt majeur de ce travail réside dans l'utilisation de jeux de données expérimentaux importants obtenus dans des conditions contrôlées. Néanmoins, l'analyse a été menée sur un site d'étude complexe à étudier à la fois en raison de sa complexité intrinsèque mais aussi du fait que les simulations aient été menées sans données de calibration préalable. Par ailleurs, le site utilisé pour les essais ne présentant qu'un dénivelé faible au regard des sites étudiés en pratique, les analyses menées pourraient être complétées par des études similaires sur d'autres sites d'études, notamment sur des versants longs, ainsi que par des analyses des capacités prédictives des études trajectographiques lorsque des données de calibration préalable sont disponibles.

\section{Références bibliographiques}

Asteriou P, Tsiambaos G, 2018. Effect of impact velocity, block mass and hardness on the coefficients of restitution for rockfall analysis. International Journal of Rock Mechanics and Mining Sciences 106: 41-50.

Bourrier F, Dorren L, Nicot F, Berger F, Darve F, 2009. Toward objective rockfall trajectory simulation using a stochastic impact model. Geomorphology 110(3):68-79.

Bourrier F, Hungr O, 2013. Rockfall Engineering, ISTE-Wiley, chapitre 6 : Rockfall Dynamics: A Critical Review of Collision and Rebound Models, pp 175-209.

Bourrier F, Berger F, Tardif P, Dorren L, Hungr O, 2012. Rockfall rebound: comparison of detailed field experiments and alternative modelling approaches. Earth Surface Processes and Landforms 37(6):656-665.

Bourrier F, Toe D, Garcia B, Baroth J, Lambert S, 2020. Experimental investigations on complex block propagation for the assessment of propagation models quality. Landslides. Submitted.

Caviezel A, Demmel SE, Ringenbach A, Buhler Y, Lu G, Christen M, Dinneen CE, Eberhard LA, Von Rickenbach D, Bartelt $P, 2019$. Reconstruction of four-dimensional rockfall trajectories using remote sensing and rock-based accelerometers and gyroscopes. Earth Surface Dynamics 7(1):199-210.

Chau K, Wong R, Lee C, 1998. Rockfall problems in hong kong and some new experimental results for coefficients of restitution. International Journal of Rock Mechanics and Mining Sciences 35(4):662-663.

Dorren LKA, Berger F, Putters US, 2006. Real-size experiments and 3-d simulation of rockfall on forested and non-forested slopes. Natural Hazards and Earth System Sciences 6(1):145-153.

Garcia B, 2020. Analyse des mécanismes d'interaction entre un bloc rocheux et un versant de propagation : application à l'ingénierie. Thèse de l'Université Grenoble Alpes.

Giani GP, Giacomini A, Migliazza M, Segalini A, 2004. Experimental and theoretical studies to improve rock fall analysis and protection work design. Rock Mechanics and Rock Engineering 37(5):369-389.

Heim A, 1932. Der Bergsturz und Menschenleben, Fretz und Wasmuth, Verlag.

Hu J, Li S, Li L, Shi S, Zhou Z, Liu H, He P, 2018. Field, experimental, and numerical investigation of a rockfall above a tunnel portal in southwestern china. Bulletin of Engineering Geology and the Environment 77: $1365-1382$. 
Labiouse V, Heidenreich B, 2009. Half-scale experimental study of rockfall impacts on sandy slopes. Natural Hazards and Earth System Sciences 9(6):1981-1993.

Lambert S, Bourrier F, Toe D, 2013. Improving three-dimensional rockfall trajectory simulation codes for assessing the efficiency of protective embankments. International Journal of Rock Mechanics and Mining Sciences 60:26-36.

Spadari M, Giacomini A, Buzzi O, Fityus S, Giani G, 2012. In situ rockfall testing in new south wales, australia. International Journal of Rock Mechanics and Mining Sciences 49:84-93.

Volkwein A, Schellenberg K, Labiouse V, Agliardi F, Berger F, Bourrier F, Dorren LKA, Gerber W, Jaboyedoff $M, 2011$. Rockfall characterisation and structural protection - a review. Natural Hazards and Earth System Sciences 11:2617-2651. 